\title{
A case of mixed-type early gastric cancer with recurrence following curative endoscopic submucosal dissection for expanded indication
}

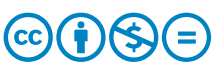

\begin{abstract}
Authors
Institutions

1 Endoscopy Division, National Cancer Center Hospital, Tokyo, Japan

2 Division of Gastroenterology and Hepatology, Toho University Omori Medical Center, Tokyo, Japan

3 Division of Pathology and Clinical Laboratories, National Cancer Center Hospital, Tokyo, Japan
\end{abstract}

Shunsuke Kobayashi ${ }^{1,2}$, Satoru Nonaka ${ }^{1}$, Ichiro Oda ${ }^{1}$, Seiichiro Abe ${ }^{1}$, Haruhisa Suzuki ${ }^{1}$, Shigetaka Yoshinaga ${ }^{1}$, Hirokazu Taniguchi ${ }^{3}$, Shigeki Sekine ${ }^{3}$, Yoshinori Igarashi², Yutaka Saito' ${ }^{1}$

submitted 25.1.2019

accepted after revision 20.3.2019

\author{
Bibliography \\ DOI https://doi.org/10.1055/a-0900-3835 | \\ Endoscopy International Open 2019; 07: E841-E845 \\ (c) Georg Thieme Verlag KG Stuttgart · New York \\ eISSN 2196-9736
}

Corresponding author

Satoru Nonaka, MD, PhD, Endoscopy Division, National

Cancer Center Hospital, 5-1-1 Tsukiji, Chuo-ku, Tokyo 1040045, Japan

Fax: $+81-3-3542-3815$

snonaka@ncc.go.jp
ABSTRACT

Background and study aims In Japan, intramucosal gastric adenocarcinoma with ulcerative finding having a predominantly differentiated type with an undifferentiated component, tumor diameter $\leq 3 \mathrm{~cm}$, and no lymphovascular invasion is included in the expanded pathological criteria for curative endoscopic treatment. This indication is based on retrospective examination of surgical resection cases, and is determined to have a negligible risk of lymph node metastasis (LNM). We performed endoscopic submucosal dissection on a 78-year-old man with early gastric cancer in 2011, and pathology revealed a well-differentiated tubular adenocarcinoma $(21 \times 10 \mathrm{~mm}$ in diameter), with poorly differentiated adenocarcinoma components, limited to the mucosa, fibrosis by ulcer scar in the submucosal layer, no lymphovascular invasion, and tumor-free margins. Resection was determined to be curative under expanded indications of the gastric cancer treatment guidelines, $4^{\text {th }}$ edition. However, 55 months after the initial diagnosis, invasive local and distant recurrence was noted. Ultimately, the patient died of gastric cancer 3 months after recurrence.

\section{Introduction}

According to version 3 of the Japanese Gastric Cancer Treatment Guidelines (JGCTG; 2010), non-curative resection by endoscopic submucosal dissection (ESD) is indicated for intramucosal, differentiated-type cancers $\leq 3 \mathrm{~cm}$ in diameter with ulceration accompanied with some areas of undifferentiatedtype histology. Takizawa et al. reported on incidence of LNM in patients with such early gastric cancer (EGC) who had undergone gastrectomy with lymph node dissection [1]. All of those patients had intramucosal adenocarcinomas of predominantly differentiated type with an undifferentiated component, ulcerative finding $(U L[+])$, tumor diameter $\leq 3 \mathrm{~cm}$, and no lymphovascular invasion, and none of the 386 patients had LNM (95\% confidence interval [CI]: $0-0.8$ ). This evidence was adopted as follows in version 4 of the JGCTG (2014): "A new rule in the current version is that if a component of undifferentiated type carcinoma was found in tumor size $\leq 3 \mathrm{~cm}$, differentiatedtype histologically, pT1a, UL(+), but was not the predominant histological type, and the endoscopic resection will be regarded as curative" [2]. In the current case, we experienced for the first time invasive recurrence of EGC following ESD, although version 4 of the JGCTG (2014) indicated that resection would be curative.

\section{Case report}

A 78-year-old man with a past history of dissecting thoracic aortic aneurysm and right cerebral hemorrhage sequelae was admitted to our hospital for treatment of early gastric cancer (EGC) in 2011. He also had left paresis, and his Eastern Cooperative Oncology Group performance status score was 2 . The 

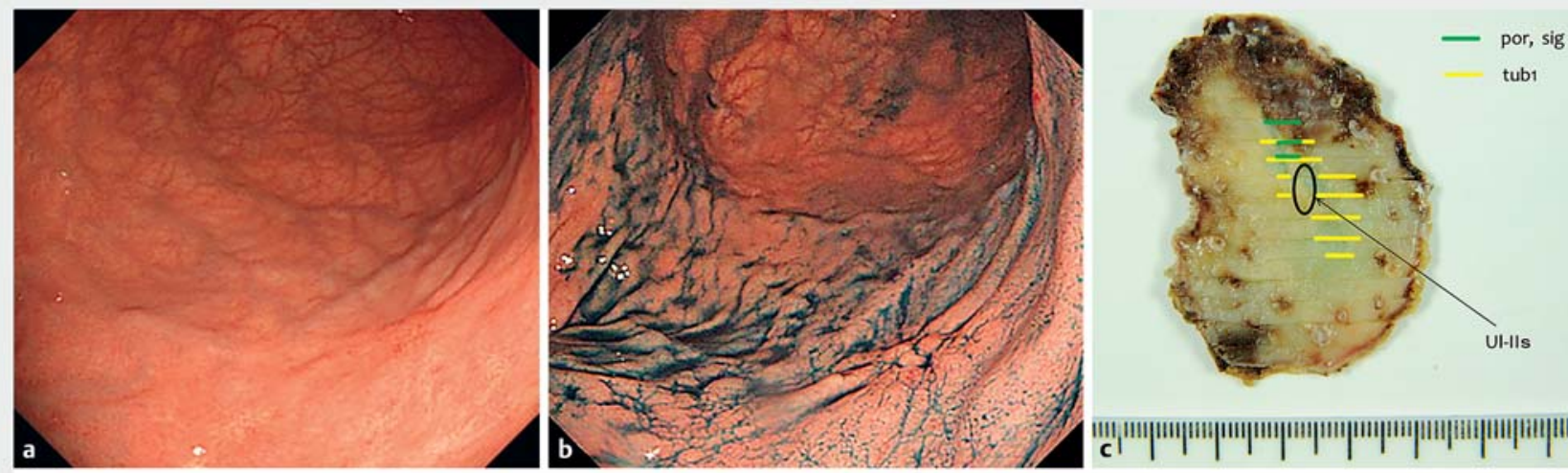

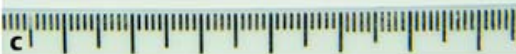
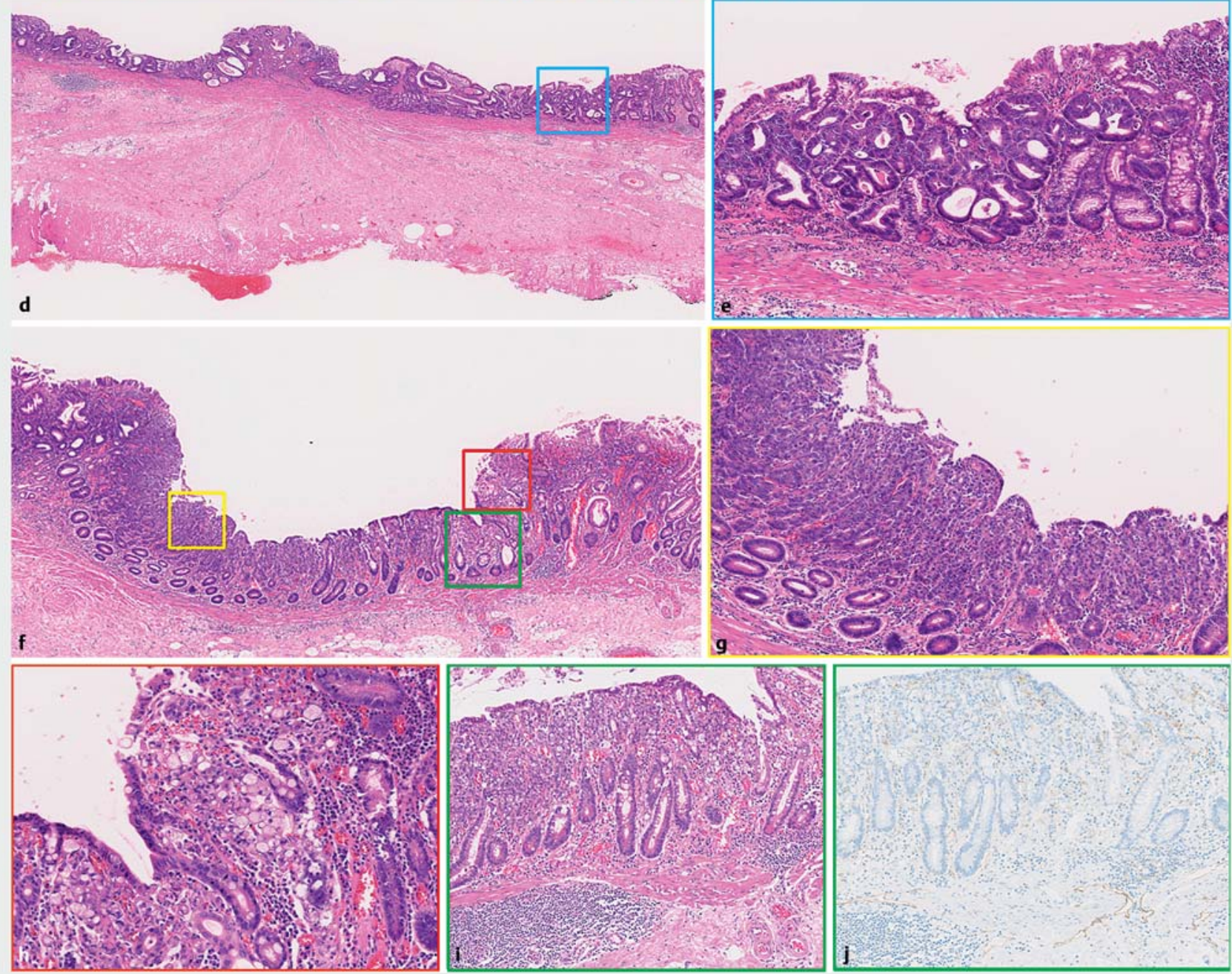

- Fig. 1 a, b Esophagogastroduodenoscopy detected a Type 0-Ilc lesion located on the posterior wall of greater curvature of the upper gastric body, limited to mucosa, $20 \mathrm{~mm}$ in size, with an ulcer scar (UL+). c, d, f Pathological examination revealed a Type 0 -IIc lesion, $21 \times 10 \mathrm{~mm}$ in size, $\mathbf{d}, \mathbf{e}$ well-differentiated tubular adenocarcinoma $\mathbf{f}, \mathbf{g}, \mathbf{h}$ with undifferentiated components, $\mathbf{d}$ fibrosis by ulcer scar in the submucosal layer, no lymphvascular invasion and tumor-free margins. $\mathbf{f}, \mathbf{i}, \mathbf{j}$ additional deeper cutting and anti-lymphatic endothelial antibodies (D2 - 40) showed no submucosal invasion or lymphatic invasion.

lesion was preoperatively diagnosed, in accordance with the Japanese Classification of Gastric Carcinoma (JCGC), as Type 0 Ilc, extending from the posterior wall to the greater curvature of the upper gastric body. It was an intramucosal lesion, $2 \mathrm{~cm}$ in diameter, with an ulcer scar (UL[+]) ( $\bullet$ Fig. $1 \mathbf{a}, \mathbf{b})$.

The biopsy specimen revealed a well-differentiated tubular adenocarcinoma, and the lesion was thought to fulfil the crite- 


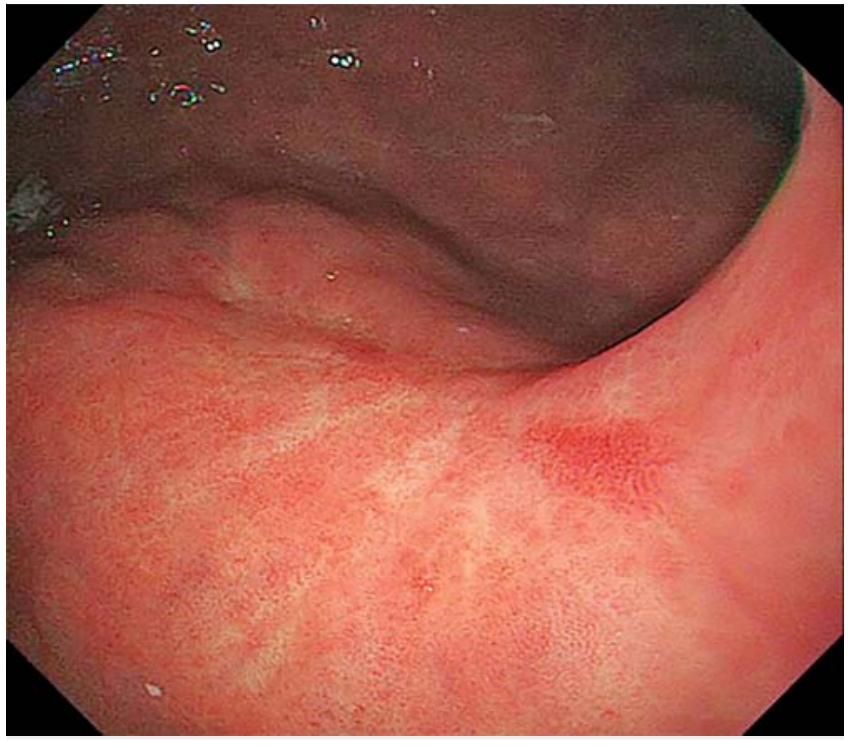

- Fig. 2 Follow-up esophagogastroduodenoscopy 43 months after gastric ESD showed only ulcer scar.

ria for expanded indications for endoscopic submucosal dissection (ESD). Thus, the lesion was subjected to gastric ESD, resulting in an en-bloc resection without any adverse events. Pathological examination, slices taken from the entire specimen at 2-mm intervals as shown in the Japanese Classification of Gastric Carcinoma, and immunohistochemical staining performed when needed revealed that the tumor was type 0 -IIc, with a 21-×10-mm diameter, a well-differentiated, intramucosal, tubular adenocarcinoma with undifferentiated components, fibrosis in the form of an ulcer scar in the submucosal layer, no lymphovascular invasion, and tumor-free margins. We evaluated the resected specimen and found an undifferentiated adenocarcinoma component based on additional deeper cutting and measurement of anti-lymphatic endothelial antibodies (D2 - 40). However, we could not find an area of tissue of

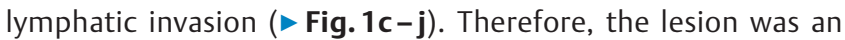
undifferentiated mixed-type cancer with an ulcer scar, and it was determined that the resection was non-curative according to version 3 of the JGCTC (2010). However, after discussion between the endoscopists, surgeons and patient, no additional surgery was performed because the patient was of advanced age and had a performance status of 2 , as well as a past history of several severe illnesses. Thus, the patient was followed up using biannual blood tests and computed tomography (CT), as well as annual esophagogastroduodenoscopy (EGD).

None of the EGD or CT examinations between 2011 and 2014 indicated any evidence of recurrence ( $\triangleright$ Fig. 2). However, 55 months after the gastric ESD, EGD demonstrated marked wall thickening in the area of the ESD scar ( $\bullet$ Fig. 3a). We diagnosed the lesion as a non-metachronous cancer because marked wall thickness was seen in the same location as the scar from the prior ESD. A biopsy specimen revealed a poorly differentiated adenocarcinoma, and the patient was diagnosed as having a recurrence after gastric ESD ( $\vee$ Fig. $\mathbf{3 b}$ ). A subse-
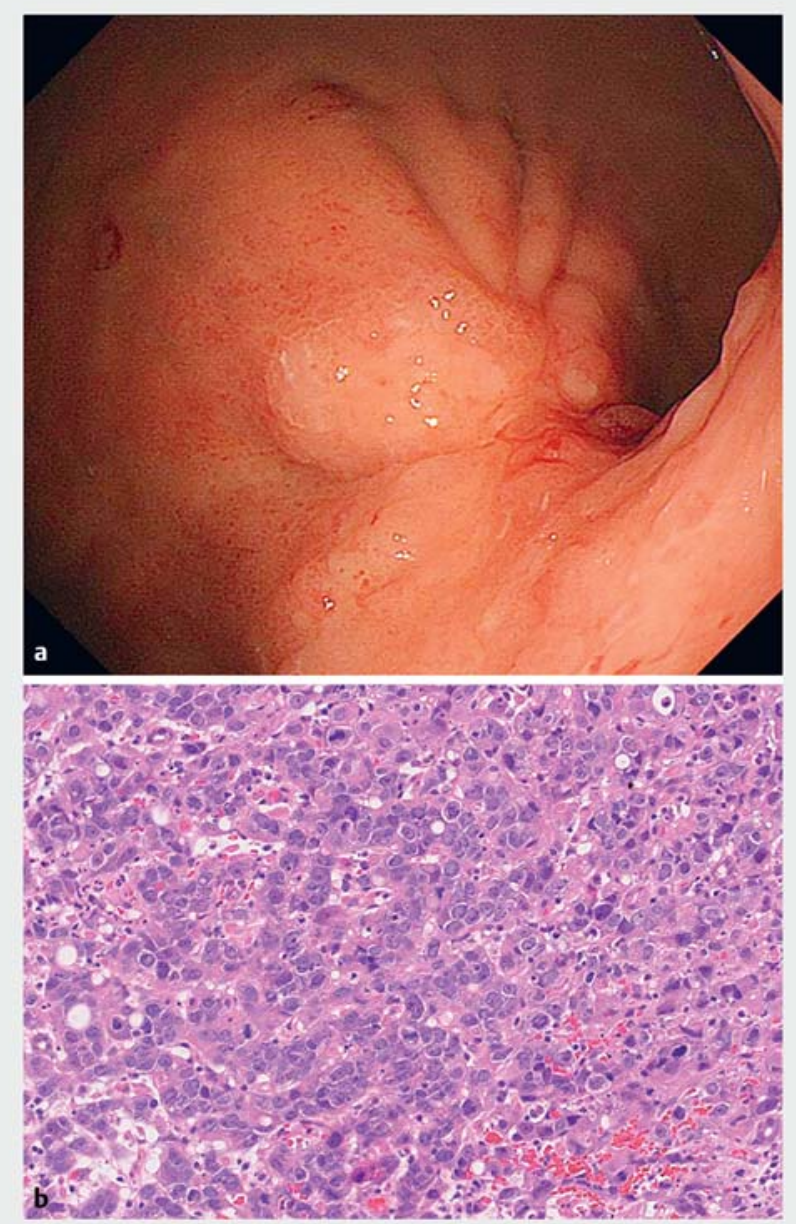

- Fig. 3 a Esophagogastroduodenoscopy 55 months after gastric ESD demonstrated the remarkable wall thickness at the ESD scar in the posterior wall of the greater curvature of the upper gastric body. $\mathbf{b}$ The biopsy specimen revealed poorly differentiated adenocarcinoma (por).

quent CT showed no regional lymph node or distant metastasis, and the status of the recurrence was thought to be cT4aNOMO, cStage IIB, according to the Japanese Classification of Gastric Carcinoma. A treatment strategy with curative intent was proposed: total gastrectomy with lymph node dissection, because the lesion was located in the upper gastric body. However, the patient refused to undergo such radical surgery. Thus, after repeated discussion between the endoscopists, surgeons, and patient, it was decided that no further treatment would be carried out-neither surgery nor chemotherapy.

One month later, the patient was urgently hospitalized in his local hospital due to a bowel obstruction and aspiration pneumonia. With immediate conservative therapy, his general condition improved, but CT showed multiple enlarged lymph nodes, both the para-aortic and intra-abdominal ( $\mathbf{F i g . 4 a , b ) . ~}$ Furthermore, the man's dietary intake was gradually getting worse, and he ultimately died of gastric cancer 3 months after the recurrence was diagnosed. 

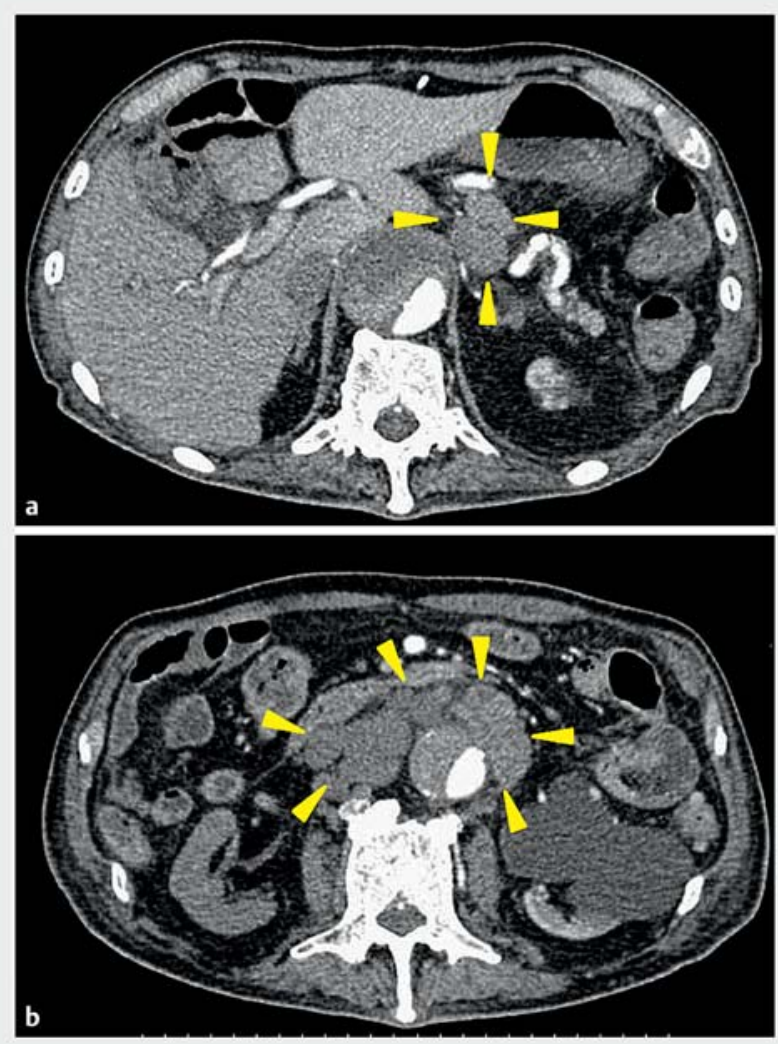

- Fig. 4 Abdominal computer tomography showed enlargement of para-aortic and intra-abdominal at 59 months after endoscopic submucosal dissection.

\section{Discussion}

Gotoda et al. found that no cases of surgical EGC without lymphovascular involvement had lymph node metastases among the following cancer types: (1) intramucosal, differentiatedtype, UL(-) cancers $>2 \mathrm{~cm}$ in diameter; (2) intramucosal, differentiated-type, $\mathrm{UL}(+)$ cancers $\leq 3 \mathrm{~cm}$ in diameter; (3) intramucosal, undifferentiated-type, UL(-) cancers $\leq 2 \mathrm{~cm}$ in diameter; and (4) superficial submucosal invasion (SM1), differentiatedtype cancers $\leq 3 \mathrm{~cm}$ in diameter. Based on this result, the expanded pathological criteria for curative endoscopic resection of EGC were developed

Subsequently, Suzuki et al. reported 5-year survival outcomes among 756 patients with EGC who had undergone curative ESD on the basis of the expanded indications between 1999 and 2006 at our institution [3]. Of the 756 patients, 212 were diagnosed as having intramucosal, differentiated, UL(+) adenocarcinoma $\leq 3 \mathrm{~cm}$ in diameter, and none of the patients experienced recurrence during median follow-up of 83.3 months. Hasuike et al. conducted a non-randomized phase II trial among 470 patients of the expanded indications for ESD in treatment of differentiated-type EGC(cT1a) with a UL(-) tumor $>2 \mathrm{~cm}$ in diameter or a $\mathrm{UL}(+)$ tumor $\leq 3 \mathrm{~cm}$ in diameter. They reported no recurrence during median follow-up of 73.8 months (range: 9.3 - 98.8 months) [4]. Thus, long-term outcomes with ESD for treatment of expanded indications for EGC are extremely good. Based on this evidence, expanded-indication lesions, such as UL $(+)$ tumors $\leq 3 \mathrm{~cm}$ in diameter, have been changed to absoluteindication lesions in version 5 of the JGCTG (2018).

In addition, five case reports of recurrence have reported after histological confirmation of curative resection in expanded-indication lesions of differentiated EGC, including two cases of histologically mixed-type cancer, two cases of SM1, differentiated-type cancer, and one case of UL(+), differentiated-type cancer [5-9]. It follows that mixed-type EGC may confer a greater risk of LNM than pure-type lesions, particularly in cases of $\mathrm{UL}(+)$ lesions. However, no studies have been limited to only mixed-type EGC, so although LNM risk is thought to be high, this is not clear.

On the other hand, Sekiguchi et al. reported that risk of LNM in 3131 surgically resected cases of EGC at our hospital [10]. In this article, there were no cases of LNM among patients with EGC without lymphovascular involvement and intramucosal pure differentiated-type, UL(-) adenocarcinoma, regardless of size ( $0 \%$; $95 \% \mathrm{Cl}: 0.0-2.9), \mathrm{UL}(+)$ adenocarcinoma $\leq 3 \mathrm{~cm}$ in diameter ( $0 \%$; $95 \% \mathrm{Cl}: 0.0-3.8)$, or $\mathrm{UL}(+)$ adenocarcinoma $>3 \mathrm{~cm}$ in diameter $(0 \% ; 95 \% \mathrm{Cl}: 0.0-2.9)$. In contrast, mixed-type adenocarcinoma with predominantly differentiated components confers a higher risk of LNM among UL(-) tumors, regardless of size (4.4\%; $95 \% \mathrm{Cl}: 1.2-10.9)$, $\mathrm{UL}(+)$ tumors $\leq 3 \mathrm{~cm}$ in size $(0 \% ; 95 \% \mathrm{Cl}: 0.0-7.0)$, and $\mathrm{UL}(+)$ tumors $>3 \mathrm{~cm}$ in size (11.4\%; $95 \% \mathrm{Cl}: 5.1-21.3)$. Takizawa et al. also reported that the rate of LNM among patients with mixed, predominantly undifferentiated-type, intramucosal gastric cancer was higher than among those with mixed, predominantly differentiatedtype cancers who had undergone surgery (24\% vs. $0 \%$ ) [1].

Undifferentiated-type cancers have more malignant potential than differentiated-type cancers. Therefore, mixed-type cancers are thought to confer a higher risk of LNM than puretype cancers. The current case was a difficult lesion to manage because the treatment results changed from non-curative resection to curative resection because guidelines had changed over time. Unfortunately, the patient died of the primary EGC recurrence. It is natural to assume that mixed, undifferentiated-type cancers and presence of UL increase the degree of malignancy. It is important to remember that risk of recurrence is not zero in lesions of the kind described here for which resection is confirmed as curative according to the limits of the criteria.

\section{Conclusion}

In conclusion, we described a fatal case of recurrence following curative resection by ESD for EGC as the expanded indication with $U \mathrm{~L}(+)$ intramucosal adenocarcinoma $\leq 3 \mathrm{~cm}$ of a predominantly differentiated type with an undifferentiated component. Although such an occurrence is believed to be rare, clinicians should still pay attention to recurrence and LNM, even when curative resection is achieved histologically. 


\section{Competing interests}

None

\section{References}

[1] Takizawa K, Ono H, Yamamoto Y et al. Incidence of lymph node metastasis in intramucosal gastric cancer measuring $30 \mathrm{~mm}$ or less, with ulceration; mixed, predominantly differentiated-type histology; and no lymphovascular invasion: a multicenter retrospective study. Gastric Cancer 2016; 19: 1144 - 1148

[2] Japanese gastric cancer treatment guidelines 2014 (ver. 4). Gastric Cancer 2017; 20: 1-19

[3] Suzuki H, Oda I, Abe $\mathrm{S}$ et al. High rate of 5-year survival among patients with early gastric cancer undergoing curative endoscopic submucosal dissection. Gastric Cancer 2016; 19: 198-205

[4] Hasuike N, Ono H, Boku N et al. A non-randomized confirmatory trial of an expanded indication for endoscopic submucosal dissection for intestinal-type gastric cancer (cT1a): the Japan Clinical Oncology Group study (JCOG0607). Gastric Cancer 2018; 21: 114-123
[5] Hanaoka N, Tanabe S, Higuchi K et al. A rare case of histologically mixed-type intramucosal gastric cancer accompanied by nodal recurrence and liver metastasis after endoscopic submucosal dissection. Gastrointest Endosc 2009; 69: 588-590

[6] Fujii $\mathrm{H}$, Ishii E, Tochitani S et al. Lymph node metastasis after endoscopic submucosal dissection of a differentiated gastric cancer confined to the mucosa with an ulcer smaller than $30 \mathrm{~mm}$. Dig Endosc 2015; $27: 159-161$

[7] Ochiai T, Ominami M, Nagami Y et al. Lymph node metastasis of mixed adenoneuroendocrine carcinoma after curative resection using the expanded criteria for early gastric cancer. Intern Med (Tokyo, Japan) 2018; 57: 2837-2842

[8] Abe S, Oda I, Nakajima T et al. A case of local recurrence and distant metastasis following curative endoscopic submucosal dissection of early gastric cancer. Gastric Cancer 2015; 18: 188-192

[9] Oya H, Gotoda T, Kinjo T et al. A case of lymph node metastasis following a curative endoscopic submucosal dissection of an early gastric cancer. Gastric Cancer 2012; 15: 221 - 225

[10] Sekiguchi M, Oda I, Taniguchi $\mathrm{H}$ et al. Risk stratification and predictive risk-scoring model for lymph node metastasis in early gastric cancer. J Gastroenterol 2016; 51: 961 -970 